\title{
Urticarial Vasculitis Successfully Treated With Omalizumab
}

\author{
Aylin Türel Ermertcan, MD; Ayşe Aktaş, MD; Ferdi Öztürk, MD; Peyker Temiz, MD
}

\section{PRACTICE POINTS}

- The differential diagnosis of urticaria and urticarial vasculitis may be complicated.

- Omalizumab is an effective urticaria treatment and also can be an alternative treatment choice in resistant urticarial vasculitis.
To the Editor:

Urticarial vasculitis (UV) is a clinicopathologic entity. It manifests as an eruption of erythematous wheals that clinically resemble urticaria, but the lesions of UV last longer, may leave residual hyperpigmentation, and may or may not be pruritic. ${ }^{1}$ Therapies most often employed include oral antihistamines and systemic immunosuppressant drugs such as corticosteroids, dapsone, colchicine, or hydroxychloroquine. ${ }^{2}$ We present a woman with UV who successfully was treated with omalizumab.

A 49-year-old woman presented to our outpatient clinic with generalized pruritic skin rashes of 2 years' duration. She also described swelling on the upper eyelids 2 times monthly. She used several antihistamines (up to 4 times daily) and was taking systemic corticosteroids and antidepressants. Physical examination revealed generalized erythematous and edematous papules and plaques on the trunk and extremities (Figure 1). At follow-up a few days later, we observed that the lesions were lasting for more than 24 hours, but there was no residual pigmentation. According to clinical concerns and the association with angioedema, we initially thought the diagnosis was chronic urticaria and angioedema. The patient had
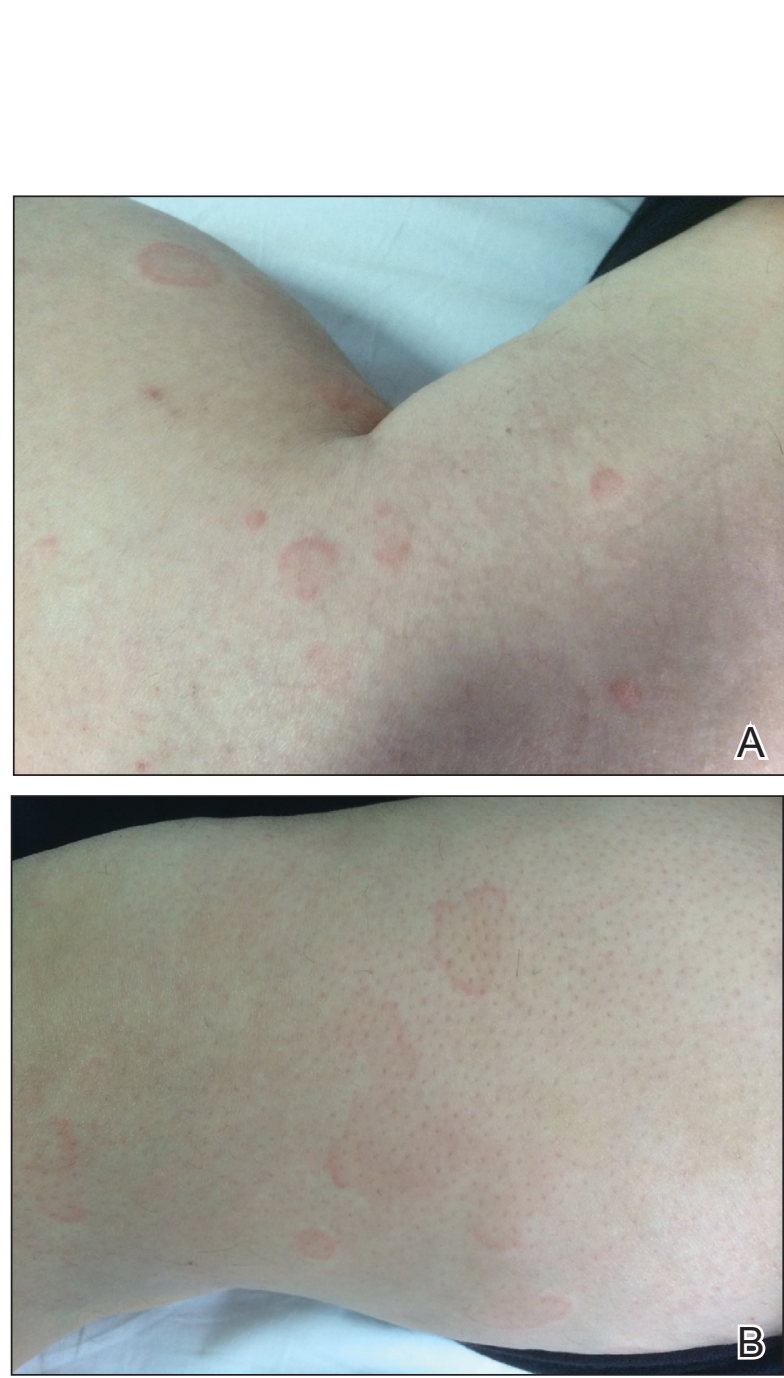

FIGURE 1. A and B, Erythematous and edematous annular papules and plaques on the arms and legs, respectively.

Dr. Ermertcan is from the Department of Dermatology; Dr. Aktaş is from the Department of Allergy and Immunology; and Dr. Temiz is from the Department of Pathology, Manisa Celal Bayar University, Turkey. Dr. Öztürk is from the Department of Dermatology, Bursa Uludağ University, Turkey. The authors report no conflict of interest.

Correspondence: Aylin Türel Ermertcan, MD, Manisa Celal Bayar University, Faculty of Medicine, Department of Dermatology, 45010 Manisa, Turkey (draylinturel@hotmail.com).

doi:10.12788/cutis.0272 
no extracutaneous manifestations such as fever, arthralgia, or lymphadenopathy. Routine laboratory examinations including antinuclear antibodies were within reference range. She had normal C3 and C4 levels and an elevated total IgE level (344 IU/mL [reference range, 0-170 IU/mL]). Because the IgE level was elevated and she had no response to the highest dosages of antihistamines, we decided to start omalizumab therapy. Prior to starting omalizumab, we performed a skin biopsy for histopathologic and direct immunofluorescence examinations for UV, as the duration of the lesions was more than 24 hours. Histopathologic examination revealed lymphocytes within the vessel wall and perivascular lymphocytic infiltration with eosinophils (Figure 2). On direct
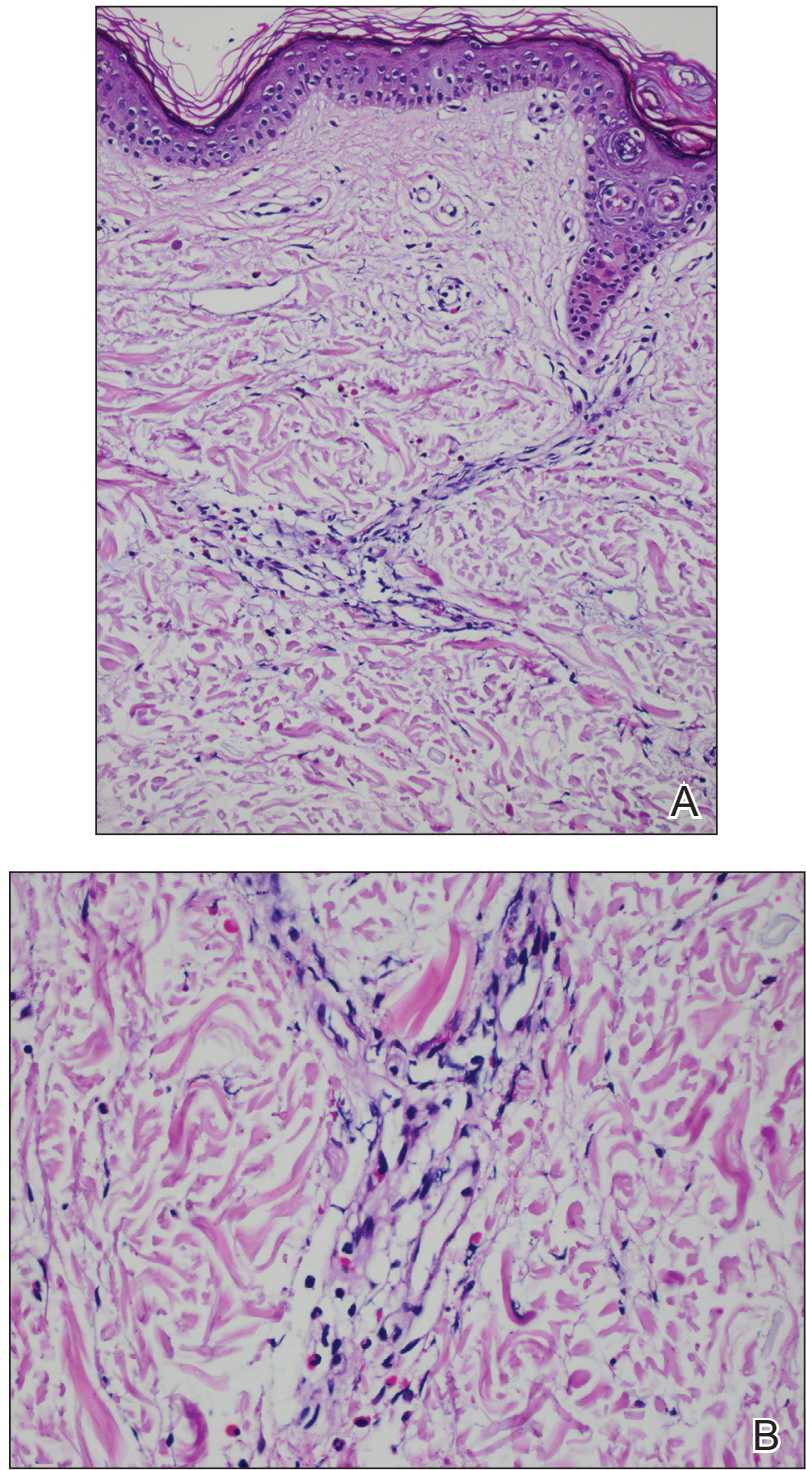

FIGURE 2. A and B, Histopathology showed lymphocytes and eosinophils within the vessel wall $(H \& E$, original magnifications $\times 100$ and $\times 200)$. immunofluorescence, perivascular IgA deposition was observed (Figure 3). Histopathologic findings were associated with lymphocytic vasculitis. Systemic involvement was not detected on detailed laboratory and radiologic examinations.

After the first application of omalizumab, the lesions disappeared within a few days. She was treated with subcutaneous omalizumab $300 \mathrm{mg}$ every 4 weeks for 6 months, and we did not observe any adverse effects related to the drug. There was no relapse after therapy cessation.

Omalizumab is a recombinant humanized anti-IgE monoclonal antibody that is approved by the US Food and Drug Administration for treatment of chronic idiopathic urticaria. ${ }^{3-5}$ Studies have suggested that omalizumab might play an important role in the treatment of other potentially IgE-mediated disease processes including allergic asthma, atopic dermatitis, allergic rhinitis, nasal polyposis, and severe ocular allergies. ${ }^{6}$ The proposed mechanism of action of omalizumab includes reduction of free IgE through the reversible formation of tiny, biologically inert complexes; targeting IgEexpressing B cells; and inhibiting production of IgE. Because it reduces free IgE, omalizumab has been used in normal IgE or hyper-IgE situations. Omalizumab also induces eosinophil apoptosis; increases IL-2, IL-3, tumor necrosis factor $\alpha$, and IFN- $\gamma$; and reduces IL-4. ${ }^{7}$ A number of off-label uses have been described such as atopic dermatitis, bullous pemphigoid, hyper-IgE syndrome, cutaneous mastocytosis, toxic epidermal necrolysis, and eosinophilic granulomatosis with polyangitis. ${ }^{8}$ There are no clinical studies of omalizumab for UV, and only a few case reports have shown that omalizumab also might be beneficial for this condition. ${ }^{2-4}$ Diez et $\mathrm{al}^{4}$ reported 3 cases of women aged 28,51, and 54 years with spontaneous chronic urticaria with autoimmune and pressure components as well as vasculitis whose symptoms

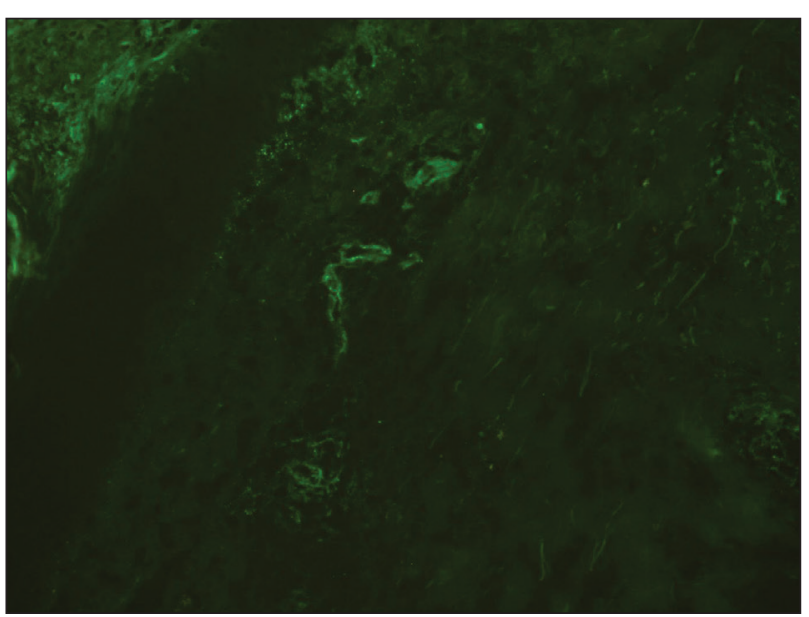

FIGURE 3. Direct immunofluorescence revealed perivascular IgA deposition (original magnification $\times 200$ ). 
completely improved after starting omalizumab. Kai et al ${ }^{3}$ successfully treated a patient with normocomplementemic UV with omalizumab and suggested that omalizumab markedly improved the patient's quality of life with chronic urticaria and UV. Ghazanfar and Thomsen ${ }^{2}$ reported the case of a 68-year-old man diagnosed with histopathologically confirmed leukocytoclastic vasculitis. He had used systemic corticosteroid therapy and dapsone without notable improvement. The patient was switched to subcutaneous omalizumab 300 mg once every 4 weeks; after 1 month, he observed complete remission of the UV and symptoms. ${ }^{2}$

Our case suggest that omalizumab has a beneficial effect on patients with UV. Omalizumab may be effective in UV through its reduction of IgE, as in chronic urticaria, and through downstream effects on cellular activation mechanisms (possibly a reduction in chemotaxis or immune complex formation). However, the mechanism of action of omalizumab for UV remains, in part, unresolved. It is not known whether omalizumab is efficacious against both normocomplementemic and hypocomplementemic UV. Further studies with a greater number of patients are needed to confirm the effects of omalizumab for vasculitic patients.

\section{REFERENCES}

1. Chang S, Carr W. Urticarial vasculitis. Allergy Asthma Proc. 2007; 28:97-100.

2. Ghazanfar MN, Thomsen SF. Omalizumab for urticarial vasculitis: case report and review of the literature. Case Rep Dermatol Med. 2015:576893.

3. Kai AC, Flohr C, Grattan CE. Improvement in quality of life impairment followed by relapse with 6-monthly periodic administration of omalizumab for severe treatment-refractory chronic urticaria and urticarial vasculitis. Clin Exp Dermatol. 2014;39:651-652.

4. Diez LS, Tamayo LM, Cardona R. Omalizumab: therapeutic option in chronic spontaneous urticaria difficult to control with associated vasculitis, report of three cases. Biomedica. 2013;33:503-512.

5. Maurer M, Rosen K, Hsieh HJ. Omalizumab for chronic urticaria. N Engl J Med. 2013;368:2530.

6. Ben Shoshan M. Omalizumab: not only for asthma. Recent Pat Inflamm Allergy Drug Discov. 2008;2:191-201.

7. Fueyo-Casado A, Campos-Munoz L, Gonzalez-Guerra E, et al. Effectiveness of omalizumab in a case of urticarial vasculitis. Clin Exp Dermatol. Published March 1, 2017. doi:10.1111/ced.13076

8. Chia JC, Mydlarski PR. Dermatologic uses of omalizumab. I Dermatol Treat. Published November 7, 2016. doi:10.1080 /09546634.2016.1249819 